\title{
"In VR, everything is possible!": Sketching and Simulating Spatially-Aware Interactive Spaces in Virtual Reality
}

\author{
Hans-Christian Jetter* \\ Christoph Anthes*
}

Roman Rädle ${ }^{\dagger}$
Judith Friedl*

Tiare Feuchtner $^{\dagger}$

*University of Applied Sciences Upper Austria, Hagenberg Campus, Austria ${ }^{\dagger}$ Aarhus University, Denmark

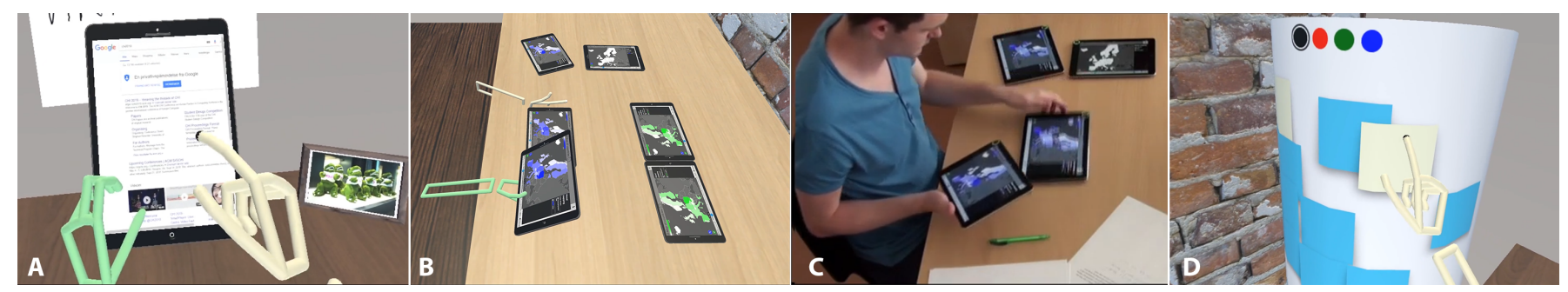

Figure 1. We created a virtual environment for designers, in which they can generate and arrange an arbitrary number of devices that execute realworld web applications (A). This allows simulation of existing interactive spaces and multi-device systems (B, C) [71], as well as sketching of new interactions with diverse tracking systems or futuristic devices, e.g., a cylindrical touch screen (D).

\begin{abstract}
We propose using virtual reality (VR) as a design tool for sketching and simulating spatially-aware interactive spaces. Using VR, designers can quickly experience their envisioned spaces and interactions by simulating technologies such as motion tracking, multiple networked devices, or unusual form factors such as spherical touchscreens or bezel-less display tiles. Design ideas can be rapidly iterated without restrictions by the number, size, or shape and availability of devices or sensors in the lab. To understand the potentials and challenges of designing in VR, we conducted a user study with 12 interaction designers. As their tool, they used a custom-built virtual design environment with finger tracking and physics simulations for natural interactions with virtual devices and objects. Our study identified the designers' experience of space in relation to their own bodies and playful design explorations as key opportunities. Key challenges were the complexities of building a usable yet versatile VR-based "World Editor".
\end{abstract}

\section{Author Keywords}

Interactive Spaces; Spatial Awareness; Interaction Design; Virtual Reality; Design tools.

\section{CCS Concepts}

-Human-centered computing $\rightarrow$ Systems and tools for interaction design; Ubiquitous and mobile computing design and evaluation methods;

Permission to make digital or hard copies of all or part of this work for personal or classroom use is granted without fee provided that copies are not made or distributed for profit or commercial advantage and that copies bear this notice and the full citation on the first page. Copyrights for components of this work owned by others than ACM must be honored. Abstracting with credit is permitted. To copy otherwise, or republish, to post on servers or to redistribute to lists, requires prior specific permission and/or a fee. Request permissions from permissions@acm.org.

CHI '20, April 25-30, 2020, Honolulu, HI, USA.

(C) 2020 Association of Computing Machinery.

ACM ISBN 978-1-4503-6708-0/20/04 _..\$15.00.

http://dx.doi.org/10.1145/3313831.3376652

\section{INTRODUCTION}

Interactive spaces are ubiquitous computing environments that integrate multiple connected post-WIMP ${ }^{1}$ devices in settings such as meeting rooms, visualization labs, control rooms, or design studios [45]. They combine devices such as interactive walls [33], tabletops [89], or mobile devices like tablets and smartphones $[34,54,73]$, which are connected via custombuilt applications that enable data sharing and collaboration. The design of interactive spaces is an ongoing research topic in HCI but remains challenging because it requires a focus on interaction and visualization techniques, as well as on the physical environment, spatial layout, and form factors of devices [45]. Interactions and spatial design must both fit the users' tasks, workflows, and ergonomic requirements [45], or they must be sufficiently configurable to adapt to changing user needs [55]. Hence, recent research has focused on spatial-awareness, allowing interactive spaces to react to configurations and movements of users and devices in a meaningful manner by means of proxemic $[56,57]$ or spatially-aware interactions [21, 73, 74, 93]. Ideally, interaction with such spaces is facilitated through familiar spatial actions instead of complex commands [74], and thus feels "natural" [45].

Given the lack of tools and methods for testing multi-device experiences [22] and, especially, spatially-aware interactive spaces, designers currently need to set up motion tracking systems $[47,56,57,72,73,94]$ and juggle many different devices and display configurations $[34,55,95]$ during distributed development with experimental tools $[14,15,27,38,40,60$, $61,62,80]$. Prototypes must be tested with multiple physical devices (e.g., using six [71] or even more than ten tablets [34]) and for different form factors, e.g., large walls [49], tabletops $[33,88,95]$, curved or spherical displays $[8,86,92]$.

\footnotetext{
${ }_{1}^{1}$ post-WIMP = diverging from the traditional "window, icon, menu, pointing device" interaction style of personal computers [43]
} 
To address some of these challenges, we propose a design approach that uses VR as a tool for sketching and simulating spatially-aware interactive spaces. VR enables designers to rapidly create simulations of existing and futuristic spaces, devices, and sensors. These can then be virtually experienced, without the need for physically building or acquiring them. Designers can thereby easily populate arbitrarily large spaces with advanced technologies such as bezel-less display tiles, interactive walls and tabletops, spherical touchscreens, or different motion trackers. Design ideas can rapidly be iterated, without restrictions in the number, size, and shape of available devices or sensors in the lab.

During a typical four-phase user-centered design cycle (e.g. UNDERSTAND, DESIGN, PROTOTYPE, EVALUATE [36, 91]), our proposed approach is most relevant for the phases of DESIGN and PROTOTYPE. During the DESIGN phase, VR supports "sketching user experiences" in the sense of Buxton [12] by letting designers rapidly and inexpensively create, elaborate, and test many concurrent design ideas during early ideation. This helps to achieve global design maxima as opposed to only iterative improvements. Using VR, designers can turn the physical environment into a domain of design [45] by rapidly changing room sizes and shapes and placing arbitrary numbers of devices of different shapes and sizes therein. Designers can also use physical locomotion in VR to experience ergonomics, visibility, and reachability without requiring room- or wall-sized physical mockups or mock devices. During the PROTOTYPE phase, VR saves designers from needing physical lab space and setting up many different devices, e.g., up to 75 screens [49]. Instead, designers can test and experience their apps in VR on different virtual devices and screens of arbitrary sizes, numbers, or shapes. Designers can also test spatially-aware interactions without physically installing sensors and tracking systems by using the motion data from off-the-shelf VR headsets and controllers.

The goal of this paper is to understand the technological feasibility of our proposed approach, and how it could affect design practices. After discussing related work, we therefore first identify key technological challenges and solutions to contribute answers to the following research questions by describing the requirements and solutions that we applied in our technical implementation of a custom-built experimental VRbased design tool: RQ1 - "What are promising key technologies and architectures for a VR-based tool to design spatiallyaware interactive spaces?". RQ2 - "Can a VR-based tool convincingly simulate relevant aspects of interactive spaces to facilitate their design (e.g., arbitrary numbers, shapes, and sizes of devices and different motion tracking systems)?" After this, we identify key opportunities and challenges of our proposed approach based on our observations from a user study with 12 interaction designers who used our experimental tool to complete different design exercises. By this, we contribute answers to RQ3 - "How do designers use and experience an experimental VR-based design tool for interactive spaces in early design phases? What opportunities and challenges can be observed?". Our results can guide future work on VR-based design tools for spatially-aware interactive spaces.

\section{RELATED WORK}

There is a wide range of $\mathrm{HCI}$ and VR publications related to our research. Here, we focus our discussion on three main topics: (1) VR-based design tools and design processes, (2) tools for designing multi- and cross-device interactive spaces, and (3) tools for proxemics or spatially-aware interactions.

\section{VR-based Design Tools and Design Processes}

We differentiate between design and prototyping tools that were created for designing AR or VR user experiences (e.g., $[31,32,35,80])$, and design tools that enable designing user experiences of interactive products within AR/VR environments (i.e., AR/VR-based design tools). While the former is a well-researched field, the latter has not yet been extensively explored and is therefore the main focus of our research.

3D design with VR: Digitally mediated design processes have a long tradition in HCI research, e.g., providing PC-based tools for quickly creating and manipulating architectural 3D models during conceptual design [67]. In contrast to such a 2D user interface, presenting designers with stereoscopic 3D models in a virtual environment (VE) allows them to experience their designs from a first-hand perspective [17] and can enhance their experiential understanding of the metrics of designed spaces [42]. Experiencing immersive VR by wearing a head mounted display (HMD) leads to a high degree of presence [79], making users feel like they are experiencing a place instead of a story. This leads to behavior that is strongly adjusted to the VE. For example, if the virtual world is experienced as an everyday place (e.g., an office), a high level of immersion can lead users to exhibit expectations and behavior that are typically associated with that place. This makes immersive VR a powerful tool for the design and evaluation of simulated spaces. Immersion also differentiates 3D modeling in VR from modeling in outdoor environments using AR [70]. In contrast to VR, AR remains spatially-situated in real-world environments.

Sketching and rapid prototyping in VR: According to Buxton, sketching is of particular importance for designers and has for centuries been the archetypical activity of design [12]. Tools like HoloSketch $[19,20]$ introduced sketching into VR, an approach that was evaluated by Arora et al. [2]. However, existing VR sketching tools focus primarily on static 3D modeling and not on sketching and manipulating interactions or transitions, which are key activities when designing interactive spaces. In other work, VR-based digital mock-ups (DMUs) and virtual prototypes (VPs) [84] are used to complement or replace rapid prototyping [26]. Astheimer et al. [3, 4] designed two early prototyping systems for industrial applications. Based on these, Dai et al. [18] describe three resulting use cases for the automotive industries in which CAD data and simulation results were visualized. VPs are also used in product design (e.g., for washing machines [10]), or in architecture and interior design prototyping (e.g., for hospital rooms [23]). However, the goal of that work was not the design or simulation of a product's interactive behavior, but solely its physical characteristics.

Simulating interactions in VR: More related to our work is the research of Jayaram et al. [44] who used VR to let engineers 
simulate and interactively experience the assembly process of a product to support the conceptual design phase and ensure "producibility." They applied physics simulations during the virtual assembly tasks, to enable reaching studies and other ergonomic evaluations. Gomes de Sa and Zachmann [28] similarly employ physics simulations during the verification of assembly and maintenance processes in VR. Furthermore, An et al. [1] explore collaborative interaction design of a vehicle prototype in VR including haptics. They enable designers to sketch the vehicle's interior and contours of instruments or displays, from within and outside the VE. However, the interactive behavior, functionality, and application logic of displays are not directly integrated into the VE and need to be provided externally by a Wizard of Oz. VR technology has also been used to simulate interactive AR systems, for example to overcome current technological restrictions of AR HMDs by simulating a much wider field of view [76]. Toolkits like ExProtoVAR $[68,69]$ reconstruct parts of the real environment and augment them inside a VE, to sketch and evaluate AR ideas and interaction techniques. The approach of using VR to simulate AR environments to experiment and evaluate their usability has also been proposed by Ragan et al. [75].

Design fiction in VR: Finally, VR has also been used to create immersive design fiction, for example by McVeigh-Schultz et al. [58]. In their work, speculative interactions were embedded within a VR story world and shown to industrial designers and engineers, to let them experience novel technologies for ideation and collaboration. In contrast to our work, this research focuses on story-driven, embodied experiences that are prepared in advance. Our goal is to let designers create own designs and develop own stories of use ad hoc within the VE.

\section{Tools for Multi- and Cross-Device Interactive Spaces}

Multi- and cross-device interactive spaces are an ongoing research topic in HCI ranging from early explorations such as PARC's Pads and Liveboards [85], i-Land [82], or Sentient Data Access [25], to middleware implementations (e.g., Shared Substance [27]), or user studies of fully operational prototypes like NiCE [33] and WeSpace [88]. The vast majority of design tools for multi- and cross-device interactive spaces are targeted at developers, allowing them to distribute interfaces between devices programmatically, or use special authoring tools [60]. From a designer's perspective, the necessary tools (e.g., software frameworks [6, 27, 46]) are often too difficult to use for quickly exploring design ideas or understanding important technological, design, and social challenges [29].

Dong et al. [22] identified three key challenges for creating useful, usable, and delightful multi-device experiences: 1.) the difficulty in designing the interactions between devices, 2.) the complexity of adapting interfaces to different platform UI standards, and 3.) the lack of tools and methods for testing multi-device user experiences. Different tools have therefore attempted to narrow the gap between design and development. For example, XDStudio [63] is a GUI builder designed to support interactive development of cross-device web interfaces. It allows for "simulated authoring" (i.e., designing for a multi-device environment on a single device by simulating other target devices) and "on-device authoring" (i.e., designing on the target devices themselves). In subsequent work, Nebeling et al. [61] have created cross-device browsers that enable non-technical users to distribute single-device web interfaces to multiple devices, including semi-automatic techniques for page segmentation and distribution [60]. These can be used to elicit multi-device designs from end-users and also to experience these as a designer. Weave [14] is a framework for scripting cross-device wearable interactions in JavaScript and easy-to-use high-level APIs. Expanding on that, Chi et al. [15] further support developers by analyzing scripts and automatically generating visual illustrations of step-by-step cross-device interactions as storyboards. Therefore, their target audience could potentially include technically versed designers. WatchConnect by Houben et al. [38] also supports designers and developers in prototyping cross-device applications that use the watch as both input device and output display. Finally, SurfaceConstellations by Marquardt et al. [55] allows designers to explore the ergonomic qualities of multi-device interactions. It provides a modular hardware platform for linking multiple mobile devices to easily create novel cross-device workspace environments. It includes a comprehensive library of 3D-printed link modules to connect and arrange tablets into new workspaces, and capacitive connector links between tablets for automatic recognition of connected devices.

While the afore-mentioned tools share our goal of supporting designers and developers, our approach is fundamentally different in that we move the design process from real-world 2D screens into a VE. Thereby, we can transcend the need for physically providing displays, or spatial settings and can simulate an arbitrary number of devices, including futuristic and speculative interactions.

\section{Tools for Proxemics and Spatially-Aware Interactions}

Another important stream of research are tools for making devices in interactive spaces spatially-aware $[54,73,74,93]$ and enabling proxemic interactions [56, 57]. This requires combinations of sensor hardware and processing software, based on different sensing technologies such as depth sensing cameras [94], radio triangulation [54, 57], ultra-sound [47], IR reflectance of mobile screens [73], polarized light [72], or marker-based tracking with IR cameras [56, 81, 93]. The development, setup, calibration, and maintenance of these tracking systems is typically time consuming, especially since the vast majority is based on experimental prototypes from research labs and only few are available as open source (e.g., $[56,73,94])$.

A VR-based simulation environment on the other hand, can rely on the body, head, and finger tracking capabilities of a commercial VR setup, to let users explore and interact with the VE. The user's tracked movements can be transformed into arbitrary coordinate systems and data formats, so that they can be fed into spatially-aware applications, without revealing that the sensor data is simulated. The VR environment therefore serves as a kind of meta-layer for integrating and simulating different virtual tracking systems. 


\section{DESIGN AND IMPLEMENTATION}

In order to answer RQ1 \& RQ2, we identified five essential requirements (Req1-Req5) for our design and implementation, which a VR-based design tool for spatially-aware interactive spaces should satisfy in order to support the DESIGN and PROTOTYPE phase of a user-centered design process. These requirements must be considered as addition to commonlyknown and self-evident requirements for VR systems, such as fast stereoscopic rendering and low-latency tracking to avoid motion sickness, as well as natural navigation in space by head and body movements. We formulated our requirements based on our previous experience from designing interactive spaces, spatially-aware systems, and cross-device interactions [45, 72, $73,74,95]$. The resulting Req1-Req5 aided us in choosing technologies, system components, and architectures, and in designing the user interface of our experimental tool.

Furthermore, we chose Unity $3 \mathrm{D}^{2}$ as a development platform for our VR design tool, since it is an approachable and popular development environment with good performance characteristics. The experimental tool was developed and tested on a desktop PC with an Intel i7 3.6 GHz CPU, an Nvidia GeForce GTX 1070 GPU, and 32 GB RAM. We share our source code as open source on $\mathrm{GitHub}^{3}$.

\section{Req1: Direct Manipulation of Apps and Objects}

During initial experimentation and informal user tests with an early prototype of our tool, we quickly realized that, ideally, all objects and content in the VE should support direct manipulation without complex mappings of commands to gestures or physical controllers. For example, designers should be able to interact with the simulated app on a simulated device by natural multi-touch interaction, as if they were using a real-world device. They should also be able to grab or move simulated devices using hand and body movements.

In our first prototype, we implemented interactions in the VE using Vive controllers. However, especially designers with no prior VR experience felt that interacting with virtual devices and objects felt cumbersome using the controllers and asked for more direct interaction methods. For this reason, we attached a Leap Motion ${ }^{4}$ sensor to the HMD, which allows hand and finger tracking in line of sight (Figure 2). To allow for real-time animation of virtual proxy hands in VR, Leap Motion provides Orion ${ }^{5}$ as an integration for Unity3D. For the HMD we chose an HTC Vive ${ }^{6}$ that provides precise $6 \mathrm{DoF}$ tracking of head movements and therefore allows for natural navigation of the scene through physical locomotion. This was important to us for two reasons: (i) we could avoid constraining users to one location and limiting interactions to their immediate surroundings, and (ii) since we are trying to mimic real-world scenarios, we did not want to rely only on navigation techniques such as teleportation, which have no real-world analogue.

\footnotetext{
${ }^{2}$ Unity3D version 2017.4.10f1 - https://unity3d. com

${ }^{3}$ GitHub Repo https://github . com/raedle/WeiserInWonderland

${ }^{4}$ Leap Motion https: //www . leapmotion. com

${ }^{5}$ Leap Motion Orion https://developer . leapmotion . com/orion/

${ }^{6}$ HTC Vive https: //www . vive. com/
}

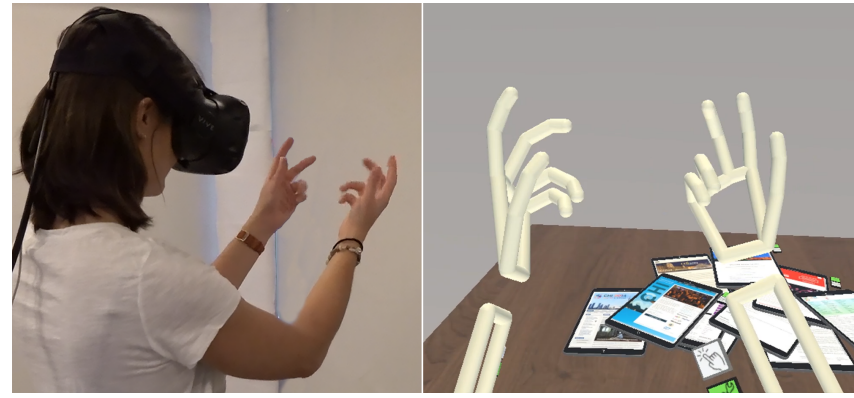

Figure 2. Our experimental design tool uses finger and hand tracking to let users naturally interact with devices and objects in the VE.

In our resulting experimental tool, users can use proxy hands to manipulate virtual objects. This includes interactions with virtual touch screen devices including free-hand drawing on surfaces. Users can pick up objects by grasping them, whereby the grasp gesture is recognized as a closing of the hand with the fingers curling in towards the thumb. To compensate for the lack of tactile feedback we indicate a successful grasp by changing the color of the proxy hand to light green. Once an object is held in hand, it can be moved around like in the real world. Opening the hand releases the grasp and the proxy hand returns to its neutral color.

While our natural hand and finger tracking lowers the threshold for beginners and reduces the number of interaction devices, its tracking fidelity is not perfect. Since the Leap Motion is an optical sensor, it can only track hands in line of sight, meaning that users can only grasp objects while they are looking at them in VR. Furthermore, it cannot track a hand when it is occluded (e.g., when one hand occludes the other or other physical objects are between sensor and hand). Unfortunately, the occlusion issue also renders the use of haptic proxies for hand-held objects impractical.

\section{Req2: Integration of Interactive Components}

In our proposed approach, VR is used to support sketching and simulating spatially-aware interactive spaces, but it does not provide a fully-fledged integrated development environment (IDE) to develop and debug applications inside the VE. Obviously, well-known IDEs for desktop- or laptop-based programming and debugging are far superior to any kind of simple authoring environment that could be provided in VR with reasonable effort. Therefore, an essential requirement for a VR-based design tool is to enable designers to quickly integrate and faithfully simulate complex real-world multi-device applications that were developed using professional IDEs by integrating interactive components into the VR space.

As the web platform has become popular for the development of multi-device applications, we demonstrate this principle by implementing a mechanism to integrate interactive web browser views in VR. In our Unity implementation, we chose ZenFulcrum's embedded browser ${ }^{7}$, which can be mapped onto any mesh, including spheres, capsules, and cylinders, as a texture (i.e., MeshFilter).

\footnotetext{
${ }^{7}$ ZenFulcrum Browser https: //zenfulcrum . com/browser
} 


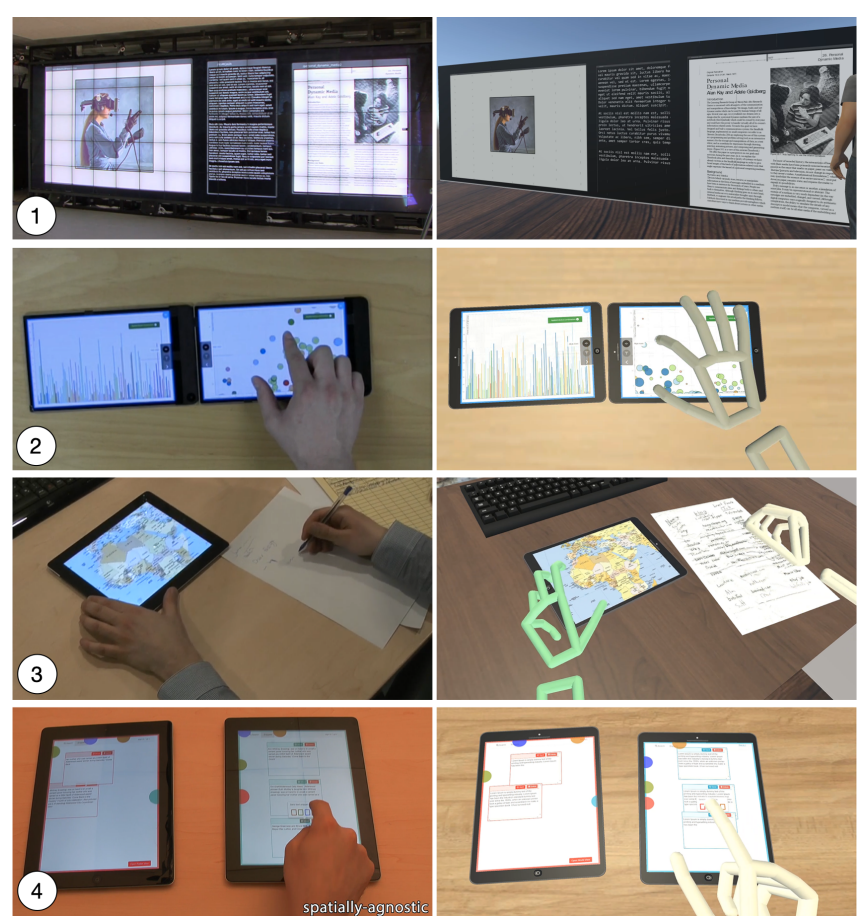

Figure 3. Simulations of existing systems in VR (right) and the original system (left). 1) Window Manager by Klokmose et al. [49] for 75 LCD tiles. 2) VisTiles by Langner et al. [51]. 3) Peephole navigation with HuddleLamp by Rädle et al. [73]. 4) Edge Bubbles by Rädle et al. [74].

To demonstrate the feasibility and scalability of this approach, we simulated the browser-based Window Manager by Klokmose et al. [49] (Figure 3.1). For this purpose, we virtually recreated the wall-sized multi-display environment consisting of 75 LCD tiles, which Klokmose et al. use for distributing applications on a very large screen. In VR, each tile is a display surface with a full-screen browser with $960 \times 960$ pixels resolution. The tiles are arranged in a grid of $15 \times 5$, resulting in an overall resolution of $14,000 \times 4,800$ pixels. This simulation of a browser-based window manager on a large interactive wall required no code changes in the original application and no further implementation within Unity, except for programmatically assigning URLs to each of the 75 tiles.

\section{Multi-Touch Support}

Although VR browsers are readily available, achieving a natural multi-touch interaction with these browsers (see Req1) requires additional implementation. Out of the box they support only single-pointer interactions with virtual interaction devices (i.e., a VR controller). Web browsers on real-world physical devices, however, support a much wider range of interactions beyond single mouse clicks or touches, such as multi-touch gestures. We therefore extended the browser component within Unity to support the PointerEvent API and the TouchEvent API to allow for multi-touch input. We then detect touch contact points in a four-step process: First, we added a game object to each fingertip, using the hand attachments included in the LeapMotion Orion library. Second, each of these game object gravitates a touch proxy by applying velocity and angular velocity to it. Thereby, the touch proxy underlies the physics of the VE. This ensures that, when users reach through a virtual browser, the touch proxy stays on the browser's surface. Also, each touch proxy has a trigger collider component. Third, each browser has a defined interaction range. For example, a planar browser has a box collider component that defines a volume above the browser surface, within which touches are registered. Fourth, when a touch proxy moves within of a browser's interaction range at runtime, we calculate the distance to the nearest point on the browser's mesh. We then trigger different browser events based on this distance (i.e., pointer\{enter,out,down,up,move\} events).

\section{Req3: Simulation of Spatially-Aware Tracking Systems}

Proxemic interactions [56, 57] and spatially-aware crossdevice interactions $[21,51,73,74,93]$ are gaining popularity in interactive spaces. They require a motion tracking system to track the location and orientation of users and devices, e.g., OptiTrack $^{8}$, Vicon ${ }^{9}$, or custom tracking systems from HCI literature $[47,56,57,72,73,94]$.

In our implementation, we simulate two spatial tracking systems: OptiTrack and HuddleLamp [73]. OptiTrack is a commercially available tracking system, used in many spatiallyaware cross-device research projects. HuddleLamp originated as a research prototype and is publicly available on GitHub. We implemented the OSC streaming protocol for the first, and the HuddleLamp server protocol for the latter. In Unity, both tracking systems are implemented as MonoBehaviors that can be attached to a box collider (on a Cube Gameobject), which serves to define the tracking volume. Each tracking simulator includes the option of enabling noise to simulate jitter, as well as adjusting the tracking frequency to match a real-world tracking system.

We demonstrate the feasibility of this by simulating two systems by Rädle et al., which both rely on HuddleLamp tracking: peephole navigation [73] (Figure 3.3) and the spatially-aware "Edge Bubbles" technique for cross-device object movement [74] (Figure 3.4). Further, we simulated VisTiles by Langner et al. [51], which uses OptiTrack and the OSC streaming protocol to react to the spatial arrangement of devices (Figure 3.2). Our implementation of the OSC streaming protocol allowed us to recreate VisTiles in VR without any modifications to its source code.

\section{Req4: Real-World and Futuristic Devices}

One of the main benefits of VR is that it frees designers from the physical limitations of the real world. For instance, designers can create large-scale interactive surfaces without having to consider issues of manufacturing, transportation, or limited lab space (see for example Figure 3.1). Designers can also experiment with non-planar or organically-shaped touchscreens, which are difficult to produce or buy today, but may be widely available in the future.

Being free to move beyond flat, rectangular displays, designers can explore novel forms of interaction [8]. For example, in our implementation for the user study we integrated a cylindrical touch screen to navigate Google Maps and a spherical touch

\footnotetext{
${ }^{8}$ NaturalPoint OptiTrack http: //optitrack. com/

${ }^{9}$ Vicon Tracker https://www . vicon. com/
} 


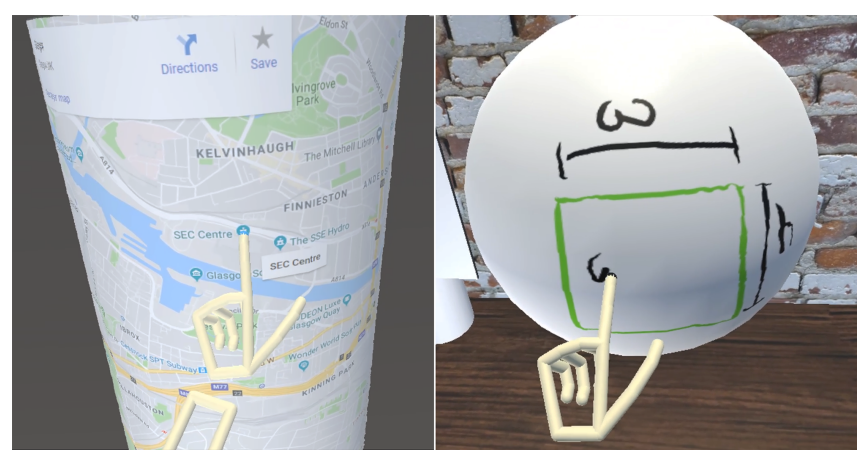

Figure 4. VR-based design tools can enable designers to experience futuristic touchscreen devices, e.g., Google Maps on a cylindrical touchscreen or drawing on a spherical display.

screen as a canvas for sketching, simply by mapping a browser onto the mesh surfaces (see Figure 4).

\section{Req5: Close Integration of Redesign and Testing}

A VR-based design tool for interactive spaces must provide designers with close cycles of redesign and testing within a single environment. When testing, the VE can be used to simulate the design and spatial configuration of devices and applications, to mimic the end-user's experience. For redesigning, the VE enables direct manipulation of the interactive space by letting designers create, move, or resize devices and objects, e.g., to attach new screens to walls or add further tablets on a meeting table. When using the VE, we differentiate two modes of interaction: use mode and design mode. We consider a close integration of both modes a requirement for successful iterative design. In our implementation, we therefore let users switch between modes using two buttons on the inside of each forearm (Figure 5, A and B).

In design mode (Figure 5, A), we allow users to configure the $\mathrm{VE}$ with displays of different shapes, as well as real-world and abstract objects. This mode specifically allows for three actions: users can (i) scale objects, (ii) turn the gravity of any object on and off, and (iii) de/activate an object's collision detection, which determines whether it can be grasped. Two buttons on each object toggle the gravity and collision status. Objects can be scaled by grasping them with both hands; when pulling the hands apart it increase in size (as if stretching the material) and pushing the hands together makes it smaller. In the current implementation, objects are scaled uniformly.

In use mode (Figure $5 \mathrm{~B}$ ), only objects with collision status turned on remain graspable and can be rescaled, or moved around in virtual space. Furthermore, the browsers on all devices are interactive. When looking at one's palm, a menu with a color palette appears. Users can choose colors by dipping their finger tips into the colored orbs, and can turn their finger tip into an eraser by touching the cube. This allows users to engage in activities like drawing on a web canvas using their fingers.

\section{RQ1 \& RQ2: Summary and Results}

Based on our experiences from building our experimental implementation of a VR-based design tool and from simulating existing spatially-aware applications inside this VE, we conclude the technological feasibility of building a VRbased design tool that satisfies Req1-Req5 with off-the-shelf devices and software tools (RQ1). In particular, the use of browsers in VR to integrate complex real-world functionality has proven very useful and reduces the need for code changes or (re)implementation of applications. This enabled us to realistically simulate complex distributed applications in an interactive virtual space with up to 75 display tiles and two different motion tracking systems (RQ2).

\section{USER STUDY}

To identify and understand the opportunities and challenges of sketching and simulation of interactive spaces in VR, we conducted a user study with 12 interaction designers ( 7 female, 5 male; mean age 32.6, SD 4.2). Our goal was to observe how they would work with our experimental VR-based design tool, and to let them comment on what it felt like to use it and how it affected their design process. While we only invited designers with substantial experience in interaction design, we did not exclude people without prior experience in designing interactive spaces or using VR.

All participants P1-P12 had received professional training in interaction design and possessed 2 to 12 years of practical design experience (mean 6.5, SD 3.4). They also reported 1 to 15 years of experience with application development (mean 9.5, SD 5.8), 0 to 7 years of experience with multi-device interaction (mean 3.1, SD 3.0), and 0 to 5 years experience with VR (mean 2.6, SD 1.8). Their degrees ranged from BSc (P6) to $\mathrm{PhD}(\mathrm{P} 4, \mathrm{P} 5, \mathrm{P} 12)$ including a professor (P12).

\section{Procedure \& Tasks}

The lab study took place on four consecutive days with three sessions per day and one participant per session. The session duration was 80.2 minutes on average (SD 10.8). Each session consisted of seven parts: (1) a brief introduction to the study, (2) completing a demographic questionnaire, (3-5) three consecutive study parts while wearing the HMD with Leap Motion finger tracking, and (6) a concluding interview followed by (7) a short debriefing. Parts $3 \& 4$ each began with the viewing of slides and videos, which showed examples of physical designs of interactive spaces and spatial-awareness from related work (part 3: [55, 73], part 4: [8]). This was intended to inspire participants for the following design tasks of growing complexity.

In part 3, the tasks served to first teach participants how to navigate the VE by physical locomotion, how to interact with virtual touchscreens in VR (including tapping links and moving sliders), how to sketch simple UI layouts on mobile devices by drawing on multiple virtual tablets with their fingers, and how to interact with non-planar displays (i.e., by panning a map on a cylindrical touchscreen and drawing the outlines of Europe and Africa on a spherical touchscreen). Part 3 concluded with allowing participants to unimanually or bimanually grab tablets and drag them out of a spawn box. This way, they could create as many tablets as desired and place them on a table with a simulated HuddleLamp for peephole navigation of a world map [73]. 


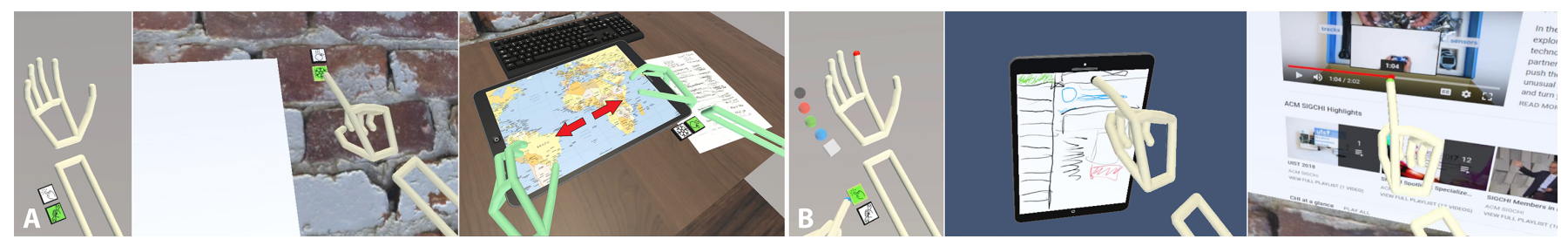

Figure 5. In design mode (A), users can manipulate device properties and resize them. In use mode (B), users can draw on devices and interact with real-world applications using (multi-)touch.

In part 4 , participants were asked to spawn a single tablet, scale it to the size of an interactive whiteboard, and attach it to a wall. To complete this, they needed to familiarize themselves with the design mode and how to deactivate gravity and collisions for selected devices. They were then asked to spawn different display shapes from multiple spawn boxes, including rectangles, cubes, cylinders, and spheres. Finally, participants were asked to use different shapes and sizes of displays to create a physical multi-screen setup for a multiplayer version of the popular game "Battleships". Besides sketching the physical setup, they were asked to use the canvas functionality to draw the visual game elements on the different displays.

This was followed by part 5 , which consisted of a single openended task: Participants were asked to use our design tool to freely create and sketch a multi-device interactive space of their choosing. We asked them to create whatever came to mind and provided a list of suggestions for potential application domains, ranging from a futuristic office workplace, to a playful installation in a public space, or a multi-display system for visual analytics.

For each part 3-5, the experimenter described the tasks to the participants and explained the interactions with the tool during the initial introduction phase. Once participants felt ready to continue, the experimenter only intervened to provide technical support, warn about potential hazards (e.g., before participants' feet got tangled in the cable of the HMD), and remind participants to think aloud, voicing their perceptions and reflections. After each part, the experimenter and a second senior researcher collected their feedback in a semi-structured interview. Throughout the entire study, a junior research associate helped with managing the process.

During the study, the participants' real-world physical movements, verbal comments, and interviews were recorded with a video camera. Additionally, their navigation in, interaction with, and changes to the VE were captured using (1) OBS Studio $^{10}$ for screen recording of the rendered scene and (2) a logging mechanism that stored the entire state of the VE every few seconds. The latter could be used during analysis: loading a logfile restored the VE to the state it was in at that specific point in time. Furthermore, all three researchers took observation notes.

\section{Data Analysis}

At the end of each day, all three researchers convened for an analysis session, during which they collected and clustered the day's observations on a large interactive whiteboard, using

${ }^{10}$ OBS Studio https: //obsproject. com/ affinity diagrams to organize ideas. Each observation was represented by a colored sticky note that carried the observation and the respective participant numbers P1-P12. The color was chosen according to the note's role (e.g., "opportunity", "challenge", "desired feature"). The process of (re)clustering and (re)coloring of notes was repeated on each of the four days, until all researchers felt that their observations were faithfully represented. In hindsight, the saturation of the clustering scheme was reached after the sixth participant. All following participants did not contribute new clusters, but added to and confirmed existing ones. At the end of the final analysis session on the last day of the study, the whiteboard contained 195 sticky notes with 4 different colors in 15 clusters. From these clusters, six were chosen, based on their relevance for the HCI community. These are reported on here, in the form of two opportunities $(\mathrm{O} 1, \mathrm{O} 2)$ and two challenges $(\mathrm{C} 1, \mathrm{C} 2)$.

\section{OBSERVED OPPORTUNITIES AND CHALLENGES}

During our analysis, we identified recurring patterns of use and how designers reacted to and interacted with our experimental prototype. In the following sections, we report these observations not to evaluate our specific implementation, but to derive more general insights about the opportunities and challenges of our proposed approach of sketching and simulating spatially-aware interactive spaces in VR. We provide these as a contribution to the HCI community and to prioritize different aspects of design and implementation for future tools.

\section{1: Experiencing Space in Relation to the Own Body}

One of the key opportunities of VR is to enable users to experience space, distance, and size within the VE from a first-hand perspective [17], and to enhance the designers' experiential understanding of the metrics of the designed spaces [42]. This first-hand experience of space in relation to the own body was typically the first aspect of the VE to be noticed by the participants, and turned out to be highly important for how they approached their designs.

\section{Natural physical locomotion inside of the design}

No participant had problems with physically moving within the VE, relying on body and head movements. For example, P7 was pleasantly surprised by the quality of the tracking of the head and hands, and how authentic moving in space felt. P3 also mentioned that moving in VR was a very natural way to experience distances (which is subject to debate [42]). Only in one case a participant reported early signs of motion sickness, but she immediately felt fine again after standing still for a few moments without taking off the HMD (P9). In some cases, participants unnecessarily avoided colliding with 
or penetrating virtual objects, such as when moving too close or inside a virtual table, since it would feel unnatural to do so (P4). Occasionally this resulted in less efficient movements by designers, but revealed a high degree of immersion.

\section{Experiencing space \& the visibility of displays}

Frequently participants were observed moving in VR to experience size relations and the visibility of displays. P3 used VR to check the visibility of objects while building "Battleships", i.e., making sure that one player really could not see the playing field of the other player. P3 built a rough sketch of a car that he referred to as a "Flintstones Car", and he squatted in the "driver's seat" to check visibility and proportions from the inside. He called this activity the "fine tuning" of displays and felt that VR greatly supports this by enabling a first person view of the design. P3, P4, P5, and P7 all mentioned that planning how a physical space should be equipped with different displays and display sizes is an important application, e.g., for deciding whether flat screens or projectors should be used (P7). P5 commented that, for this purpose future tools should also provide a greater collection of assets, including off-the-shelf displays, projectors, and mobile devices.

\section{Experiencing space \& ergonomics}

All participants were actively seeking a first-hand impression of the ergonomics of different devices; for example, the reachability of distant regions on a virtual touch screen could be explored by standing in front of the screen and trying to touch its corners. In one case, P2 reached out to check whether he could access the corners of a screen with his arm. Subsequently he bent over to reach regions that were below his waistline and therefore uncomfortable to reach. P8 used his own body height as $\mathrm{s}$ reference for judging the height and size of a display. He remarked that the tool could also help him to better experience the size of large objects (e.g., posters), which he finds too hard to predict with non-immersive design tools. P4 \& P6 both stood on their toes to reach the north pole on top of the spherical display while drawing northern Europe, and commented that it was interesting to be able to physically move around the display and experience how this would feel in reality. Furthermore, at the beginning of the study, P10 took off her high-heel shoes to be more comfortable when moving around in VR. At a later stage, however, she decided to wear them again, because they would add a few centimeters to her body height, making it easier to reach objects above her. In a similar situation, P9 jumped to reach a button above her head (Figure 6). Such experiences of different sizes or shapes of displays in relation to the own body also lead to surprising insights about device affordances and usability. For example, P3 and P9 commented that interaction with the cylindrical display felt very different when standing in front of it from what they would have expected. P9 mentioned that she would now prefer to pan the map by bimanually grasping and rotating the cylinder with her palms, instead of panning it with the tip of her index finger. P6 commented that she found it more difficult to navigate on a cylindrical screen than expected, but that the large display size helped her to not get lost.

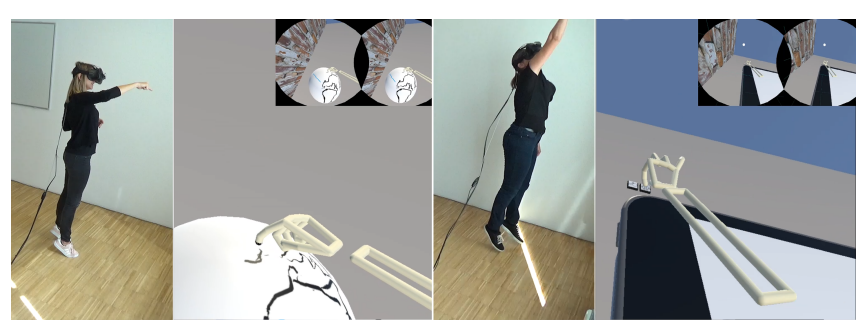

Figure 6. P6 tiptoes (left) and P9 jumps (right) to reach overhead targets.

\section{Experiencing space \& workflows}

P4 \& P9 both regarded planning of workplaces based on modular and interactive furniture as an interesting use case. P4 thought that this would be superior to 2D sketching with tools, such as Adobe Illustrator, because in VR the spatial dimensions of the design immediately becomes clear. However, she felt that simulating the interactive behavior of smart furniture in the VE (e.g., changing color or display content based on proximity or touch interaction) would be "overkill" and she would only use VR for the spatial layout and not for the design of interactions. In contrast, some participants remarked that they found it important not to regard space as separate from the tasks and workflows. Especially P7 emphasized that the integration of web applications into VR was extremely important, because otherwise he would not be able to judge display configurations and reachability in the context of a real-world workflow, e.g., to assess whether a certain room and screen design was really working for a meeting or brainstorming scenario. He would only be able to judge whether the necessary head, body, or arm movements for looking at objects or accessing tools was compatible with the task at hand, if the real applications were present. He also stated that he would have liked to use such a tool for some of his past projects. Furthermore, P7 felt reminded of the design process of the "Frankfurt Kitchen", which was designed by Schütte-Lihotzky in 1926 to optimize the spatial layout of kitchens, based on detailed time-motion studies of typical cooking workflows. P7 considered VR as a tool that could greatly help in such a design process, as is confirmed by existing VEs for kitchen or interior design, like the work by Merell et al. and Nomoto et al. $[59,65]$, or IKEA's Immerse ${ }^{11}$.

\section{O2: Playful Design Explorations and Storytelling}

According to Bill Buxton [12], stories and, more importantly, story-telling and play, are a critical part of design. We observed all three elements actively practiced during the design process and well supported by the VR technology.

\section{Playing with objects and devices}

VR invited designers to "play" with the available devices and objects in their environment. In initial phases of interaction, "playing" did not serve to tell a story or to act out a scenario, but simply to get a feel for the purpose different objects or devices could serve within a design, and to explore different ideas, spatial configurations, and sizes. Only after this initial exploration did participants begin to focus on distinct application domains or scenarios. For example, P7 \& P9 commented

\footnotetext{
${ }_{11}$ https://blog.siggraph. org/2018/07/

ikea-immerse-real-time-live.html/
} 
on their first steps during the free design exercise with "I don't know yet where this leads" (P7) and "it happens as it comes" (P9). After placing objects in space and viewing them from different sides, the participants then developed further ideas and explored them by experimenting with display positions and sizes.

In the case of P2 \& P9, experimenting with unusual displays reshaped their design goals. They found it inspiring that they could draw on the cube display and also rotate it with their hands. P9 chose a cube display as the center of her "Battleships" design to create a four-player instead of a two-player game. P2 designed a rotatable display cube with one application per side for remotely controlling a large screen. After finishing his design, P7 commented that the result was not what he originally had in mind, but he now found it more intriguing. We believe that the immediate visual and spatial feedback inside the VE invited designers to question their ideasm and to develop new ideas based on altering the current state. The VE therefore supports what Buxton describes as the spirit of design [12]: to invite, to suggest, and to question. It also enables an iterative creative process that Buxton refers to as a "dialogue with a sketch": Creating a sketch from existing design knowledge and then creating new design knowledge by "reading" (or experiencing) the resulting sketch.

A further benefit of the VE was to support the designers' imagination. P6 and P9 both commented that their imaginative power was greatly increased in the VE, compared to a 2D sketch. P5 mentioned that the VE invites scribbling and thinking about futuristic ideas, and suggested to also use it for designing holograms or 3D visualizations, since "in VR, everything is possible!". As one example of an imaginative design, P1 created a playful environment in which small spherical displays could be thrown at a planar display to get points. Again, "play" was an important aspect here, since this idea arose from P1 throwing a display sphere into the air with his hands and catching it again (Figure 7).

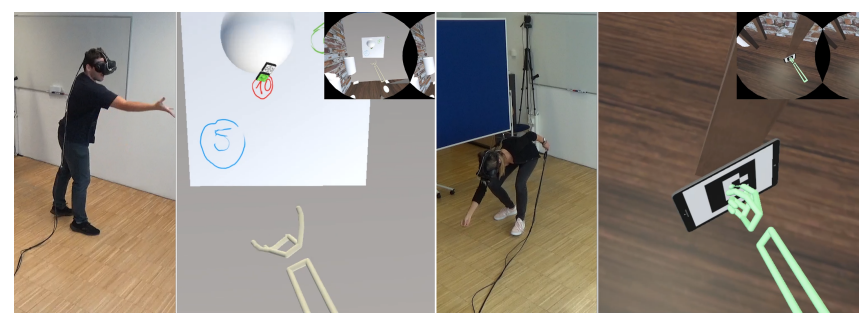

Figure 7. P1 throwing a ball at the targets on the display (left). P6 picking up a tablet she dropped (right).

\section{Playing or "acting out" in VR}

Sketching user experiences requires visual storytelling to fill the gaps between the different states of a system with transitions (e.g., interactions, animations, shifts of user focus) [12]. The necessary techniques of UX sketching (e.g., storyboarding, animations [12]) require much experience, especially for multi-device or cross-device designs. A similar challenge is representing the context of use and user intentions with techniques to "play" them or "acting out", such as using scenarioor acting-type approaches in ideation [12, 41].
During our study, we observed that designers used VR as a backdrop for storytelling about users and their interactions. Instead of creating multiple instances of a design to document different states and transitions between them, they preferred to explain their designs by using natural physical movements or actions within the VE. For example, when explaining the result of the free design exercise, they explained the layout and the role of objects and devices. This included informally describing or acting out interactions and user's intentions, navigating through space, directing the attention of their audience (i.e., the experimenters) by visually focusing on or pointing at different virtual devices, leaving marks or highlights with the drawing functionality, or grabbing objects to carry them from one place to another. P7 commented that the VE had shifted his focus towards storytelling and workflows and drew parallels to LEGO's Serious Play methodology, which is applied, for example, to teach software engineering and help people brainstorm and discuss complex ideas through storytelling and metaphors [50]. The VE was therefore not only used to create physical layouts and sketches of screen content, but also to actively demonstrate the envisioned user interactions. For future designs of VR-based design tools, it is therefore desirable to include functions that can facilitate, record, and replay such demonstrations.

\section{C1: No "One-size-fits-all" Design Tools}

During the study, we also identified challenges that designers faced when sketching and simulating in VR. The first challenge (C1) concerns the fidelity of the simulation: it is unlikely that a VE will ever be able to cover all relevant design aspects of interactive spaces. There is too wide a breadth of design ideas and necessary tools, ranging from wireframing of wallsized data visualizations to simulating low-level pen input techniques on a spherical display. Therefore, designers will always need to use different tools to make their specific design ideas visible and measurable (see Lim et al.'s discussion of the anatomy of prototypes and their role in design [52]). P2, P5 \& P7 commented that the design tool and the set of available assets influenced their results, because some of their ideas were tedious or impossible to explore with the given setup. P7 elaborated that for some questions he had faced during previous projects the VE would have been very helpful. However, he added that it would not have been helpful at all in others, such as for testing "micro interactions" (P7), like how different stylus tips or pen gestures felt on different interactive surfaces. We therefore believe that VR-based design tools for interactive spaces will need specialized and purpose-built components for exploring and testing different aspects of a design. It is therefore unlikely that a single VR-based design tool could serve as a "one-size-fits-all" solution, but it could provide a platform or framework for the integration of specific components.

\section{C2: The Complexities of Building a "World Editor"}

All participants welcomed the possibility of directly manipulating and editing their environment in VR, while still being able to interact with the applications on the devices. Creating, scaling, or moving displays and devices proved essential for the design process. They enjoyed doing this directly with their 
hands, using their virtual "superpowers" for moving, throwing, or scaling devices, and switching off gravity. P9 repeatedly exclaimed "cool!" when creating new tablets and placing them in mid-air, after previously witnessing how devices can fall to the ground (Figure 7). After not paying attention to the experimenters' comments for a short period, P9 excused herself for having "so much fun" interacting in the VE. P7 felt reminded of the 2007 short film "World Builder" by Bruce Branit ${ }^{12}$, in which a man builds a holographic world for his comatose wife to let her virtually experience a beloved place.

However, the overall usability of our experimental design tool still showed much room for improvement. We chose a minimal set of functionalities that merely included the simplest variants of typical interactions. For example, we only enabled uniformscaling of objects and did not provide any mechanisms for precisely positioning or aligning objects. There was also no delete, copy, or paste function. Furthermore, having to switch between "use" and "design" mode confused some participants. Nonetheless, the menus and buttons that appeared on the user's palm and on the inside of the forearm were very well received (P1,P2,P6,P7,P9,P10).

Similar to previous work on the usability of VR or AR [7, 48, 53], we observed that the interaction design for a VR-based design tool is a very challenging task. It is therefore necessary to carefully consider many design and implementation choices, such as whether to use controllers vs. finger tracking, how to implement object movement and manipulation in VR based on previous research on $2 \mathrm{D}$ or AR interfaces [67, 83, 90], how to best support sketching in VR [2], how to integrate alignment and snapping mechanisms $[9,64,66]$ or mode switching for system control [48], and how to deal with the absence of the sense of touch $[11,77,78,87]$. These efforts should not be underestimated.

\section{RQ3: Summary of User Study Findings}

Based on our study, we identified the experience of space in relation to the own body, and the support of playful design explorations and storytelling as key opportunities of our proposed approach. Two main challenges are the lack of "onesize-fits-all" design tools in VR that will satisfy all needs of designers, and the difficulty of designing the tool itself due to the complexities of building a "world editor".

\section{LIMITATIONS AND FUTURE WORK}

We observed a number of challenges in our work, as described in the previous section (in particular C2). From these we identified several limitations of our research that could be addressed in future work.

(1) Vision-based hand-tracking: Using an optical sensor for hand tracking allows users to interact without wearing a glove. However, this also entails several drawbacks. Firstly, the lack of haptic feedback makes it harder for users to know when a virtual object is touched or grabbed. While in our prototype this was partially compensated by visual feedback, future technological solutions could further improve on this by including wearable actuators, ranging from simple vibration motors

12 https : / / www : youtube . com/watch?v=QP3YywgRx 5A
$[24,87]$ to exoskeletons [30, 37], physical proxies [5, 13], or mid-air haptic feedback [39]. Secondly, with this technology, interaction is only possible while the user's hand is within the limited range of the hand tracking sensor attached to the HMD. This hinders eyes-free interaction. While this could be solved by using gloves for tracking, future research could integrate approaches that extend the optical tracking space, supporting interaction with uninstrumented hands even when they are not in the user's line of sight [16]. (2) Display resolution: In contrast to the high resolutions of real-world mobile devices, the resolutions of current VR HMDs are not sufficient to render small fonts on virtual devices as legible text inside the VE. This will likely be solved in next generation of HMDs ${ }^{13}$, which will use eye tracking for foveated rendering to create the illusion of a high-resolution VE. (3) Supporting a wider range of assets and deformable or wearable devices: Future studies and tools should provide designers with more assets in the VE, such as off-the-shelf devices including wearables that can be attached to the users' bodies. Also, letting designers deform devices in VR could reveal further design opportunities. (4) Collaboration: Interactive spaces are often designed for collaboration. Future VR-based design tools could benefit from being collaborative themselves, e.g., multiple designers could work together during design, or play the roles of different users during simulation. (5) Task load: Simulations of interactive spaces in VR do not fully reflect our physical reality (e.g., unrealistic physics, non-tangible objects). This requires designers to first learn the techniques and rules for interacting with the VE. For example, they need to discover how to use their virtual multi-purpose hands or switching between modes for transforming virtual objects and interacting with simulated applications. In our study, we therefore informally observed an increased task load (see C2) but further research is required to understand the differences between performing a design task with traditional methods or in VR. This research can then inform a better interaction design for VR-based design tools.

\section{CONCLUSION}

In this paper, we proposed using VR as a design tool for sketching and simulating spatially-aware interactive spaces. We discussed potential benefits of our proposed approach for designers and demonstrated its technological feasibility by formulating five key requirements and sharing how we implemented them in an experimental design tool using stateof-the-art VR hard- and software. Furthermore, we conducted a user study with 12 interaction designers to better understand the opportunities and challenges of our proposed approach for design practice. Our study identified the designers' experience of space in relation to their own bodies and playful design explorations as key opportunities. Key challenges were the complexities of building a usable yet versatile VR-based "World Editor".

\section{ACKNOWLEDGEMENTS}

This work was partially funded by Aarhus Universitets Forskningsfond, the Innovation Fund Denmark (MADE Digital project, IFD grant no. 6151-00006B), and the USIVIS project of the Austrian Research Promotion Agency (FFG).

\footnotetext{
13 https: //varjo.com/
} 


\section{REFERENCES}

[1] Sang-Gyun An, Yongkwan Kim, Joon Hyub Lee, and Seok-Hyung Bae. 2017. Collaborative Experience Prototyping of Automotive Interior in VR with 3D Sketching and Haptic Helpers. In Proceedings of the 9th International Conference on Automotive User Interfaces and Interactive Vehicular Applications (AutomotiveUI '17). ACM Press, Oldenburg, Germany, 83-192. DOI : http://dx.doi.org/10.1145/3122986.3123002

[2] Rahul Arora, Rubaiat Habib Kazi, Fraser Anderson, Tovi Grossman, Karan Singh, and George Fitzmaurice. 2017. Experimental Evaluation of Sketching on Surfaces in VR. In Proceedings of the 2017 CHI Conference on Human Factors in Computing Systems (CHI '17). ACM, New York, NY, USA, 5643-5654. DOI : http://dx.doi.org/10.1145/3025453.3025474

[3] Peter Astheimer, Wolfgang Felger, and Stefan Müller. 1993. Virtual design: A generic VR system for industrial applications. Computers \& Graphics 17, 6 (Nov 1993), 671-677. DOI :

http://dx.doi.org/10.1016/0097-8493(93)90116-q

[4] Peter Astheimer and Martin Göbel. 1995. Virtual Design II - an advanced VR development environment. In Eurographics. Springer Vienna, Monte Carlo, Monaco, 247-272. DOI:

http://dx.doi.org/10.1007/978-3-7091-9433-1_21

[5] Mahdi Azmandian, Mark Hancock, Hrvoje Benko, Eyal Ofek, and Andrew D Wilson. 2016. Haptic Retargeting: Dynamic Repurposing of Passive Haptics for Enhanced Virtual Reality Experiences. In Proceedings of the 2016 CHI Conference on Human Factors in Computing Systems (CHI '16). ACM, 1968-1979. DOI : http://dx.doi.org/10.1145/2858036.2858226

[6] Sriram Karthik Badam and Niklas Elmqvist. 2014. PolyChrome: A Cross-Device Framework for Collaborative Web Visualization. In Proceedings of the Ninth ACM International Conference on Interactive Tabletops and Surfaces (ITS '14). ACM, New York, NY, USA, 109-118. DOI :

http://dx.doi.org/10.1145/2669485.2669518

[7] Blaine Bell, Steven Feiner, and Tobias Höllerer. 2001. View Management for Virtual and Augmented Reality. In Proceedings of the 14th Annual ACM Symposium on User Interface Software and Technology (UIST '01). ACM, New York, NY, USA, 101-110. DOI : http://dx.doi.org/10.1145/502348.502363

[8] Hrvoje Benko, Andrew D. Wilson, and Ravin Balakrishnan. 2008. Sphere: Multi-touch Interactions on a Spherical Display. In Proceedings of the 21st Annual ACM Symposium on User Interface Software and Technology (UIST '08). ACM, New York, NY, USA, 77-86. DOI : http://dx. doi .org/10.1145/1449715.1449729

[9] Eric A. Bier. 1990. Snap-dragging in Three Dimensions. In Proceedings of the 1990 Symposium on Interactive $3 D$ Graphics (I3D '90). ACM, New York, NY, USA, 193-204. DOI : http://dx.doi .org/10.1145/91385.91446
[10] Monica Bordegoni, Francesco Ferrise, and Joseba Lizaranzu. 2011. The use of interactive Virtual Prototypes for products specification in the concept design phase. In 2011 IEEE Virtual Reality Conference. IEEE, Singapore, Singapore. DOI :

http://dx.doi.org/10.1109/vr.2011.5759466

[11] Gerd Bruder, Frank Steinicke, and Wolfgang Sturzlinger. 2013. To Touch or Not to Touch?: Comparing 2D Touch and 3D Mid-air Interaction on Stereoscopic Tabletop Surfaces. In Proceedings of the 1st Symposium on Spatial User Interaction (SUI '13). ACM, New York, NY, USA, 9-16. DOI :

http://dx.doi.org/10.1145/2491367.2491369

[12] Bill Buxton. 2007. Sketching User Experiences: Getting the Design Right and the Right Design. Morgan Kaufmann Publishers Inc., San Francisco, CA, USA.

[13] Lung-Pan Cheng, Eyal Ofek, Christian Holz, Hrvoje Benko, and Andrew D Wilson. 2017. Sparse Haptic Proxy: Touch Feedback in Virtual Environments Using a General Passive Prop. In Proceedings of the 2017 CHI Conference on Human Factors in Computing Systems (CHI '17). ACM, New York, NY, USA, 3718-3728. DOI : http://dx.doi.org/10.1145/3025453.3025753

[14] Pei-Yu (Peggy) Chi and Yang Li. 2015. Weave: Scripting Cross-Device Wearable Interaction. In Proceedings of the 33rd Annual ACM Conference on Human Factors in Computing Systems (CHI '15). ACM, New York, NY, USA, 3923-3932. DOI : http://dx.doi.org/10.1145/2702123.2702451

[15] Pei-Yu (Peggy) Chi, Yang Li, and Björn Hartmann. 2016. Enhancing Cross-Device Interaction Scripting with Interactive Illustrations. In Proceedings of the 2016 CHI Conference on Human Factors in Computing Systems (CHI '16). ACM, New York, NY, USA, 5482-5493. DOI : http://dx.doi.org/10.1145/2858036.2858382

[16] Pascal Chiu, Kazuki Takashima, Kazuyuki Fujita, and Yoshifumi Kitamura. 2019. Pursuit Sensing: Extending Hand Tracking Space in Mobile VR Applications. In Symposium on Spatial User Interaction (SUI '19). ACM, New York, NY, USA, 1-5. DOI : http://dx.doi.org/10.1145/3357251.3357578

[17] James E. Cutting. 1997. How the eye measures reality and virtual reality. Behavior Research Methods, Instruments, \& Computers 29, 1 (mar 1997), 27-36. DOI : http://dx.doi.org/10.3758/bf03200563

[18] F. Dai, W. Felger, T. Frühauf, M. Göbel, D. Reiners, and Gabriel Zachmann. 1996. Virtual Prototyping Examples for Automotive Industries. In Virtual Reality World. Computerwoche Verlag, Stuttgart, Germany.

[19] Michael F. Deering. 1995. HoloSketch: a virtual reality sketching/animation tool. ACM Transactions on Computer-Human Interaction 2, 3 (Sep 1995), 220-238. DOI : http://dx.doi .org/10.1145/210079.210087 
[20] Michael F. Deering. 1996. The HoloSketch VR sketching system. Commun. ACM 39, 5 (May 1996), 54-61. DOI : http://dx.doi.org/10.1145/229459.229466

[21] Giuseppe Desolda, Carmelo Ardito, Hans-Christian Jetter, and Rosa Lanzilotti. 2019. Exploring spatially-aware cross-device interaction techniques for mobile collaborative sensemaking. Int. J. Hum.-Comput. Stud. 122 (2019), 1-20. DOI :

http://dx.doi.org/10.1016/j.ijhcs.2018.08.006

[22] Tao Dong, Elizabeth F. Churchill, and Jeffrey Nichols. 2016. Understanding the Challenges of Designing and Developing Multi-Device Experiences. In Proceedings of the 2016 ACM Conference on Designing Interactive Systems (DIS '16). ACM, New York, NY, USA, 62-72. DOI: http://dx.doi.org/10.1145/2901790.2901851

[23] Phillip S. Dunston, Laura L. Arns, James D. Mcglothlin, Gregory C. Lasker, and Adam G. Kushner. 2011. An Immersive Virtual Reality Mock-Up for Design Review of Hospital Patient Rooms. In Collaborative Design in Virtual Environments. Springer Netherlands, 167-176. DOI : http://dx.doi .org/10.1007/978-94-007-0605-7_15

[24] Tiare Feuchtner and Jörg Müller. 2018. Ownershift: Facilitating Overhead Interaction in Virtual Reality with an Ownership-Preserving Hand Space Shift. In ACM User Interface Software and Technology Symposium (UIST '18). ACM, New York, NY, USA. DOI : http://dx.doi.org/10.1145/3242587.3242594

[25] George W Fitzmaurice, Azam Khan, William Buxton, Gordon Kurtenbach, and Ravin Balakrishnan. 2003. Sentient Data Access via a Diverse Society of Devices. Queue 1, 8 (2003), 52-62. D0I :

http://dx.doi.org/10.1145/966712.966721

[26] I. Gibson, D .Brown, S. Cobb, and R. Eastgate. 1993. Virtual Reality and Rapid Prototyping: Conflicting or Complimentary?. In 1993 International Solid Freeform Fabrication Symposium. University of Texas at Austin, 113-120. DOI: http://dx.doi.org/10.15781/t2qv3cm8t

[27] Tony Gjerlufsen, Clemens Nylandsted Klokmose, James Eagan, Clément Pillias, and Michel Beaudouin-Lafon. 2011. Shared substance: developing flexible multi-surface applications. In Proceedings of the SIGCHI Conference on Human Factors in Computing Systems (CHI '11). ACM, New York, NY, USA, 3383-3392. DOI :

http://dx.doi.org/10.1145/1978942 . 1979446

[28] Antonino Gomes de Sá and Gabriel Zachmann. 1999. Virtual Reality as a Tool for Verification of Assembly and Maintenance Processes. Computers \& Graphics 23, 3 (1999), $389-403$. DOI :

http://dx.doi.org/10.1016/S0097-8493(99)00047-3

[29] Jens Grubert, Matthias Kranz, and Aaron J. Quigley. 2016. Challenges in Mobile Multi-Device Ecosystems. CoRR abs/1605.07760 (2016). DOI : http://dx.doi.org/10.1186/s13678-016-0007-y
[30] Xiaochi Gu, Yifei Zhang, Weize Sun, Yuanzhe Bian, Dao Zhou, and Per Ola Kristensson. 2016. Dexmo: An inexpensive and lightweight mechanical exoskeleton for motion capture and force feedback in VR. In Proceedings of the 2016 CHI Conference on Human Factors in Computing Systems. ACM, 1991-1995. DOI: http://dx.doi.org/10.1145/2858036.2858487

[31] Sinem Güven and Steven Feiner. 2003. Authoring 3D Hypermedia for Wearable Augmented and Virtual Reality. In Proceedings of the 7th IEEE International Symposium on Wearable Computers (ISWC '03). IEEE Computer Society, Washington, DC, USA, 118-. http://dl . acm. org/citation. cfm?id=946249. 946908

[32] Victor Haefner. 2014. PolyVR - A Virtual Reality Authoring System. In EuroVR 2014 - Conference and Exhibition of the European Association of Virtual and Augmented Reality, Jerome Perret, Valter Basso, Francesco Ferrise, Kaj Helin, Vincent Lepetit, James Ritchie, Christoph Runde, Mascha van der Voort, and Gabriel Zachmann (Eds.). The Eurographics Association. DOI: http://dx.doi.org/10.2312/eurovr.20141343

[33] Michael Haller, Jakob Leitner, Thomas Seifried, James R. Wallace, Stacey D. Scott, Christoph Richter, Peter Brandl, Adam Gokcezade, and Seth Hunter. 2010. The NiCE Discussion Room: Integrating Paper and Digital Media to Support Co-Located Group Meetings. In Proceedings of the SIGCHI Conference on Human Factors in Computing Systems (CHI '10). ACM, New York, NY, USA, 609-618. DOI : http://dx.doi.org/10.1145/1753326.1753418

[34] Peter Hamilton and Daniel J. Wigdor. 2014. Conductor: enabling and understanding cross-device interaction. In Proceedings of the 32nd annual ACM conference on Human factors in computing systems - CHI '14. ACM Press, New York, New York, USA, 2773-2782. DOI: http://dx.doi.org/10.1145/2556288.2557170

[35] Alastair Hampshire, Hartmut Seichter, Raphaël Grasset, and Mark Billinghurst. 2006. Augmented Reality Authoring: Generic Context from Programmer to Designer. In Proceedings of the 18th Australia Conference on Computer-Human Interaction: Design: Activities, Artefacts and Environments (OZCHI '06). ACM, New York, NY, USA, 409-412. DOI : http://dx.doi.org/10.1145/1228175. 1228259

[36] Rex Hartson and Pardha S Pyla. 2012. The UX Book: Process and guidelines for ensuring a quality user experience. Elsevier.

[37] Ronan Hinchet, Velko Vechev, Herbert Shea, and Otmar Hilliges. 2018. DextrES: Wearable Haptic Feedback for Grasping in VR via a Thin Form-Factor Electrostatic Brake. In The 31st Annual ACM Symposium on User Interface Software and Technology. ACM, 901-912. DOI : http://dx.doi.org/10.1145/3242587.3242657 
[38] Steven Houben and Nicolai Marquardt. 2015. WatchConnect: A Toolkit for Prototyping Smartwatch-Centric Cross-Device Applications. In Proceedings of the 33rd Annual ACM Conference on Human Factors in Computing Systems (CHI '15). ACM, New York, NY, USA, 1247-1256. DOI : http://dx.doi.org/10.1145/2702123.2702215

[39] Thomas Howard, Maud Marchal, Anatole Lécuyer, and Claudio Pacchierotti. 2019. PUMAH: Pan-tilt Ultrasound Mid-Air Haptics for larger interaction workspace in virtual reality. IEEE Transactions on Haptics (2019). DOI :

http://dx.doi.org/10.1109/TOH. 2019.2963028

[40] Maria Husmann, Michael Spiegel, Alfonso Murolo, and Moira C. Norrie. 2016. UI Testing Cross-Device Applications. In Proceedings of the 2016 ACM International Conference on Interactive Surfaces and Spaces (ISS '16). ACM, New York, NY, USA, 179-188. DOI : http://dx.doi.org/10.1145/2992154.2992177

[41] Giulio Iacucci, Carlo Iacucci, and Kari Kuutti. 2002. Imagining and Experiencing in Design, the Role of Performances. In Proceedings of the Second Nordic Conference on Human-computer Interaction (NordiCHI '02). ACM, New York, NY, USA, 167-176. DOI : http://dx.doi.org/10.1145/572020.572040

[42] Victoria Interrante, Brian Ries, and Lee Anderson. 2006. Distance Perception in Immersive Virtual Environments, Revisited. In Proceedings of the IEEE Conference on Virtual Reality (VR '06). IEEE Computer Society, Washington, DC, USA, 3-10. DOI : http://dx.doi.org/10.1109/VR. 2006.52

[43] Robert J.K. Jacob, Audrey Girouard, Leanne M. Hirshfield, Michael S. Horn, Orit Shaer, Erin Treacy Solovey, and Jamie Zigelbaum. 2008. Reality-based interaction: a framework for post-WIMP interfaces. In Proceeding of the twenty-sixth annual CHI conference on Human factors in computing systems (CHI '08). ACM Press, New York, New York, USA, 201. DOI : http://dx.doi.org/10.1145/1357054.1357089

[44] Sankar Jayaram, Hugh I Connacher, and Kevin W Lyons. 1997. Virtual assembly using virtual reality techniques. Computer-Aided Design 29, 8 (aug 1997), 575-584. DOI : http://dx . doi .org/10.1016/s0010-4485(96)00094-2

[45] Hans-Christian Jetter, Harald Reiterer, and Florian Geyer. 2014. Blended Interaction: Understanding Natural Human-Computer Interaction in post-WIMP Interactive Spaces. Personal Ubiquitous Comput. 18, 5 (June 2014), 1139-1158. DOI : http://dx.doi.org/10.1007/s00779-013-0725-4

[46] Hans-Christian Jetter, Michael Zöllner, Jens Gerken, and Harald Reiterer. 2012. Design and Implementation of Post-WIMP Distributed User Interfaces with ZOIL. International Journal of Human-Computer Interaction 28, 11 (2012), 737-747. DOI :

http://dx.doi.org/10.1080/10447318.2012.715539
[47] Haojian Jin, Christian Holz, and Kasper Hornbæk. 2015. Tracko: Ad-hoc Mobile 3D Tracking Using Bluetooth Low Energy and Inaudible Signals for Cross-Device Interaction. In Proceedings of the 28th Annual ACM Symposium on User Interface Software \& Technology (UIST '15). ACM, New York, NY, USA, 147-156. DOI : http://dx.doi.org/10.1145/2807442.2807475

[48] Joseph J. LaViola Jr., Ernst Kruijff, Ryan P. McMahan, Doug Bowman, and Ivan P. Poupyrev. 2017. 3D User Interfaces: Theory and Practice (2nd Edition) (Usability). Addison-Wesley Professional.

[49] Clemens N Klokmose, James R Eagan, Siemen Baader, Wendy Mackay, and Michel Beaudouin-Lafon. 2015. Webstrates: shareable dynamic media. In Proceedings of the 28th Annual ACM Symposium on User Interface Software \& Technology (UIST '15). ACM, 280-290. DOI:http://dx.doi.org/10.1145/2807442.2807446

[50] Stan Kurkovsky. 2015. Teaching Software Engineering with LEGO Serious Play. In Proceedings of the 2015 ACM Conference on Innovation and Technology in Computer Science Education (ITiCSE '15). ACM, New York, NY, USA, 213-218. DOI : http://dx.doi.org/10.1145/2729094.2742604

[51] Ricardo Langner, Tom Horak, and Raimund Dachselt. 2018. VisTiles: Coordinating and Combining Co-located Mobile Devices for Visual Data Exploration. IEEE transactions on visualization and computer graphics 24 , 1 (2018), 626-636. DOI : http://dx.doi.org/10.1109/TVCG. 2017.2744019

[52] Youn-Kyung Lim, Erik Stolterman, and Josh Tenenberg. 2008. The Anatomy of Prototypes: Prototypes As Filters, Prototypes As Manifestations of Design Ideas. ACM Trans. Comput.-Hum. Interact. 15, 2, Article 7 (July 2008), 27 pages. DOI: http://dx.doi.org/10.1145/1375761.1375762

[53] Robert W. Lindeman, John L. Sibert, and James K. Hahn. 1999. Towards Usable VR: An Empirical Study of User Interfaces for Immersive Virtual Environments. In Proceedings of the SIGCHI Conference on Human Factors in Computing Systems (CHI '99). ACM, New York, NY, USA, 64-71. DOI: http://dx.doi.org/10.1145/302979.302995

[54] Andrés Lucero, Jussi Holopainen, and Tero Jokela. 2011. Pass-them-around: collaborative use of mobile phones for photo sharing. In Proceedings of the 2011 annual conference on Human factors in computing systems (CHI '11). ACM Press, New York, New York, USA, 1787. DOI: http://dx.doi .org/10.1145/1978942.1979201

[55] Nicolai Marquardt, Frederik Brudy, Can Liu, Ben Bengler, and Christian Holz. 2018.

SurfaceConstellations: A Modular Hardware Platform for Ad-Hoc Reconfigurable Cross-Device Workspaces. In Proceedings of the 2018 CHI Conference on Human Factors in Computing Systems (CHI '18). ACM, New York, NY, USA, 354:1-354:14. DOI: http://dx.doi .org/10.1145/3173574.3173928 
[56] Nicolai Marquardt, Robert Diaz-Marino, Sebastian Boring, and Saul Greenberg. 2011. The Proximity Toolkit: Prototyping Proxemic Interactions in Ubiquitous Computing Ecologies. In Proceedings of the 24th Annual ACM Symposium on User Interface Software and Technology (UIST'11). ACM, New York, NY, USA, 315-326. DOI :

http://dx.doi.org/10.1145/2047196.2047238

[57] Nicolai Marquardt, Ken Hinckley, and Saul Greenberg. 2012. Cross-device interaction via micro-mobility and f-formations. In Proceedings of the 25th annual ACM symposium on User interface software and technology (UIST '12). ACM Press, New York, New York, USA, 13. DOI : http://dx.doi.org/10.1145/2380116.2380121

[58] Joshua McVeigh-Schultz, Max Kreminski, Keshav Prasad, Perry Hoberman, and Scott S. Fisher. 2018. Immersive Design Fiction: Using VR to Prototype Speculative Interfaces and Interaction Rituals Within a Virtual Storyworld. In Proceedings of the 2018 Designing Interactive Systems Conference (DIS '18). ACM, New York, NY, USA, 817-829. DOI: http://dx.doi.org/10.1145/3196709. 3196793

[59] Paul Merrell, Eric Schkufza, Zeyang Li, Maneesh Agrawala, and Vladlen Koltun. 2011. Interactive Furniture Layout Using Interior Design Guidelines. ACM Trans. Graph. 30, 4, Article 87 (July 2011), 10 pages. DOI : http://dx.doi.org/10.1145/2010324. 1964982

[60] Michael Nebeling. 2017. XDBrowser 2.0: Semi-Automatic Generation of Cross-Device Interfaces. In Proceedings of the 2017 CHI Conference on Human Factors in Computing Systems (CHI'17). ACM, New York, NY, USA, 4574-4584. DOI : http://dx.doi.org/10.1145/3025453.3025547

[61] Michael Nebeling and Anind K. Dey. 2016. XDBrowser: User-Defined Cross-Device Web Page Designs. In Proceedings of the 2016 CHI Conference on Human Factors in Computing Systems (CHI'16). ACM, New York, NY, USA, 5494-5505. DOI : http://dx.doi.org/10.1145/2858036.2858048

[62] Michael Nebeling, Maria Husmann, Christoph Zimmerli, Giulio Valente, and Moira C. Norrie. 2015. XDSession: Integrated Development and Testing of Cross-device Applications. In Proceedings of the 7th ACM SIGCHI Symposium on Engineering Interactive Computing Systems (EICS '15). ACM, New York, NY, USA, 22-27. DOI : http://dx.doi.org/10.1145/2774225.2775075

[63] Michael Nebeling, Theano Mintsi, Maria Husmann, and Moira Norrie. 2014. Interactive Development of Cross-device User Interfaces. In Proceedings of the 32Nd Annual ACM Conference on Human Factors in Computing Systems (CHI '14). ACM, New York, NY, USA, 2793-2802. DOI : http://dx.doi.org/10.1145/2556288.2556980

[64] Rui Nóbrega and Nuno Correia. 2012. Magnetic Augmented Reality: Virtual Objects in Your Space. In Proceedings of the International Working Conference on
Advanced Visual Interfaces (AVI'12). ACM, New York, NY, USA, 332-335. DOI :

http://dx.doi.org/10.1145/2254556.2254620

[65] N. Nomoto, J. Omura, K. Awada, K. Mamura, and M. Agamachi. 1995. Kitchen planning system using kansei VR. In Symbiosis of Human and Artifact, Yuichiro Anzai, Katsuhiko Ogawa, and Hirohiko Mori (Eds.). Advances in Human Factors/Ergonomics, Vol. 20. Elsevier, 185 - 190. DOI : http://dx.doi.org/https: //doi.org/10.1016/S0921-2647(06)80032-8

[66] Benjamin Nuernberger, Eyal Ofek, Hrvoje Benko, and Andrew D. Wilson. 2016. SnapToReality: Aligning Augmented Reality to the Real World. In Proceedings of the 2016 CHI Conference on Human Factors in Computing Systems (CHI'16). ACM, New York, NY, USA, 1233-1244. DOI :

http://dx.doi.org/10.1145/2858036.2858250

[67] Ji-Young Oh, Wolfgang Stuerzlinger, and John Danahy. 2006. SESAME: Towards Better 3D Conceptual Design Systems. In Proceedings of the 6th Conference on Designing Interactive Systems (DIS '06). ACM, New York, NY, USA, 80-89. DOI : http://dx.doi.org/10.1145/1142405.1142419

[68] Thies Pfeiffer and Nadine Pfeiffer-Leßmann. 2018. Virtual Prototyping of Mixed Reality Interfaces with Internet of Things (IoT) Connectivity. $i$-com 17, 2 (Aug 2018), 179-186. DOI :

http://dx.doi.org/10.1515/icom-2018-0025

[69] Nadine Pfeiffer-Leßmann and Thies Pfeiffer. 2018. ExProtoVAR: A Lightweight Tool for Experience-Focused Prototyping of Augmented Reality Applications Using Virtual Reality. In HCI International 2018 - Posters' Extended Abstracts, Constantine Stephanidis (Ed.). Springer International Publishing, Cham, 311-318. DOI :

http://dx.doi.org/10.1007/978-3-319-92279-9_42

[70] Wayne Piekarski and Bruce H. Thomas. 2001. Tinmith-Metro: New Outdoor Techniques for Creating City Models with an Augmented Reality Wearable Computer. In Proceedings of the 5th IEEE International Symposium on Wearable Computers (ISWC '01). IEEE Computer Society, Washington, DC, USA, 31-. http: //dl . acm. org/citation. cfm?id=580581. 856546

[71] Thomas Plank, Hans-Christian Jetter, Roman Rädle, Clemens Nylandsted Klokmose, Thomas Luger, and Harald Reiterer. 2017. Is Two Enough?! Studying Benefits, Barriers, and Biases of Multi-Tablet Use for Collaborative Visualization. In Proceedings of the 2017 CHI Conference on Human Factors in Computing Systems (CHI'17). 4548-4560. DOI : http://dx.doi.org/10.1145/3025453.3025537

[72] Roman Rädle, Hans-Christian Jetter, Jonathan Fischer, Inti Gabriel, Clemens N. Klokmose, Harald Reiterer, and Christian Holz. 2018. PolarTrack: Optical Outside-In Device Tracking That Exploits Display 
Polarization. In Proceedings of the 2018 CHI Conference on Human Factors in Computing Systems (CHI '18). ACM, New York, NY, USA, Article 497, 9 pages. DOI : http://dx.doi .org/10.1145/3173574.3174071

[73] Roman Rädle, Hans-Christian Jetter, Nicolai Marquardt, Harald Reiterer, and Yvonne Rogers. 2014. HuddleLamp: Spatially-Aware Mobile Displays for Ad-hoc Around-the-Table Collaboration. In Proceedings of the Ninth ACM International Conference on Interactive Tabletops and Surfaces - ITS '14. ACM Press, New York, New York, USA, 45-54. DOI : http://dx.doi.org/10.1145/2669485.2669500

[74] Roman Rädle, Hans-Christian Jetter, Mario Schreiner, Zhihao Lu, Harald Reiterer, and Yvonne Rogers. 2015. Spatially-aware or Spatially-agnostic?: Elicitation and Evaluation of User-Defined Cross-Device Interactions. In Proceedings of the 33rd Annual ACM Conference on Human Factors in Computing Systems. 3913-3922. DOI: http://dx.doi .org/10.1145/2702123.2702287

[75] E. Ragan, C. Wilkes, D.A. Bowman, and T. Hollerer. 2009. Simulation of Augmented Reality Systems in Purely Virtual Environments. In 2009 IEEE Virtual Reality Conference. IEEE. DOI : http://dx.doi.org/10.1109/vr.2009.4811058

[76] Donghao Ren, Tibor Goldschwendt, YunSuk Chang, and Tobias Hollerer. 2016. Evaluating wide-field-of-view augmented reality with mixed reality simulation. In 2016 IEEE Virtual Reality (VR). IEEE. DOI : http://dx.doi.org/10.1109/vr.2016.7504692

[77] G. Robles-De-La-Torre. 2006. The importance of the sense of touch in virtual and real environments. IEEE MultiMedia 13, 3 (July 2006), 24-30. DOI: http://dx.doi.org/10.1109/MMUL.2006.69

[78] Samuel B. Schorr and Allison M. Okamura. 2017. Fingertip Tactile Devices for Virtual Object Manipulation and Exploration. In Proceedings of the 2017 CHI Conference on Human Factors in Computing Systems (CHI '17). ACM, New York, NY, USA, 3115-3119. DOI :

http://dx.doi.org/10.1145/3025453.3025744

[79] Mel Slater and Sylvia Wilbur. 1997. A framework for immersive virtual environments (FIVE): Speculations on the role of presence in virtual environments. Presence: Teleoperators \& Virtual Environments 6, 6 (1997), 603-616. DOI :

http://dx.doi.org/10.1162/pres.1997.6.6.603

[80] Maximilian Speicher, Brian D. Hall, Ao Yu, Bowen Zhang, Haihua Zhang, Janet Nebeling, and Michael Nebeling. 2018. XD-AR: Challenges and Opportunities in Cross-Device Augmented Reality Application Development. Proc. ACM Hum.-Comput. Interact. 2 , EICS, Article 7 (June 2018), 24 pages. DOI: http://dx.doi .org/10.1145/3229089

[81] Martin Spindler, Marcel Martsch, and Raimund Dachselt. 2012. Going Beyond the Surface: Studying Multi-layer Interaction Above the Tabletop. In
Proceedings of the SIGCHI Conference on Human Factors in Computing Systems (CHI'12). ACM, New York, NY, USA, 1277-1286. DOI:

http://dx.doi.org/10.1145/2207676.2208583

[82] Norbert A. Streitz, Jörg Geißler, Torsten Holmer, Shin'ichi Konomi, Christian Müller-Tomfelde, Wolfgang Reischl, Petra Rexroth, Peter Seitz, and Ralf Steinmetz. 1999. i-LAND: an interactive landscape for creativity and innovation. In Proceedings of the SIGCHI conference on Human factors in computing systems the CHI is the limit (CHI '99). ACM Press, New York, New York, USA, 120-127. DOI:

http://dx. doi . org/10.1145/302979.303010

[83] Robert J. Teather, Andriy Pavlovych, Wolfgang Stuerzlinger, and I. Scott MacKenzie. 2009. Effects of Tracking Technology, Latency, and Spatial Jitter on Object Movement. In Proceedings of the 2009 IEEE Symposium on $3 D$ User Interfaces (3DUI '09). IEEE Computer Society, Washington, DC, USA, 43-50. DOI : http://dx.doi.org/10.1109/3DUI. 2009.4811204

[84] G. Gary Wang. 2002. Definition and Review of Virtual Prototyping. Journal of Computing and Information Science in Engineering 2, 3 (2002), 232. DOI: http://dx.doi.org/10.1115/1.1526508

[85] M Weiser. 1991. The computer for the 21st century. Scientific American 3, 3 (1991), 94-104. DOI: http://dx.doi .org/10.1145/329124.329126

[86] Malte Weiss, Simon Voelker, Christine Sutter, and Jan Borchers. 2010. BendDesk: Dragging Across the Curve. In ACM International Conference on Interactive Tabletops and Surfaces (ITS '10). ACM, New York, NY, USA, 1-10. DOI :

http://dx.doi. org/10.1145/1936652.1936654

[87] Eric Whitmire, Hrvoje Benko, Christian Holz, Eyal Ofek, and Mike Sinclair. 2018. Haptic Revolver: Touch, Shear, Texture, and Shape Rendering on a Reconfigurable Virtual Reality Controller. In Proceedings of the 2018 CHI Conference on Human Factors in Computing Systems (CHI'18). ACM, New York, NY, USA, Article 86, 12 pages. DOI : http://dx. doi . org/10.1145/3173574.3173660

[88] Daniel Wigdor, Hao Jiang, Clifton Forlines, Michelle Borkin, and Chia Shen. 2009. WeSpace: The Design Development and Deployment of a Walk-up and Share Multi-surface Visual Collaboration System. In Proceedings of the SIGCHI Conference on Human Factors in Computing Systems (CHI '09). ACM, New York, NY, USA, 1237-1246. DOI : http://dx. doi. org/10.1145/1518701.1518886

[89] Daniel Wigdor, Chia Shen, Clifton Forlines, and Ravin Balakrishnan. 2006. Table-Centric Interactive Spaces for Real-Time Collaboration. In Proceedings of the Working Conference on Advanced Visual Interfaces (AVI'06).

ACM, New York, NY, USA, 103-107. DOI : http://dx.doi.org/10.1145/1133265.1133286 
[90] Graham Wilson, Mark McGill, Matthew Jamieson, Julie R. Williamson, and Stephen A. Brewster. 2018. Object Manipulation in Virtual Reality Under Increasing Levels of Translational Gain. In Proceedings of the 2018 CHI Conference on Human Factors in Computing Systems (CHI '18). ACM, New York, NY, USA, Article 99, 13 pages. DOI:

http://dx.doi.org/10.1145/3173574.3173673

[91] John R. Wilson. 1999. Virtual environments applications and applied ergonomics. Applied Ergonomics 30, 1 (Feb 1999), 3-9. DOI :

http://dx.doi.org/10.1016/s0003-6870(98)00040-4

[92] Raphael Wimmer, Fabian Hennecke, Florian Schulz, Sebastian Boring, Andreas Butz, and Heinrich Hußmann. 2010. Curve: Revisiting the Digital Desk. In Proceedings of the 6th Nordic Conference on Human-Computer Interaction: Extending Boundaries (NordiCHI '10). ACM, New York, NY, USA, 561-570. DOI : http://dx.doi .org/10.1145/1868914.1868977

[93] Paweł Wozniak, Nitesh Goyal, Przemysław Kucharski, Lars Lischke, Sven Mayer, and Morten Fjeld. 2016.
RAMPARTS: Supporting Sensemaking with Spatially-Aware Mobile Interactions. In Proceedings of the 2016 CHI Conference on Human Factors in Computing Systems (CHI '16). ACM, New York, NY, USA, 2447-2460. DOI :

http://dx.doi.org/10.1145/2858036.2858491

[94] Chi-Jui Wu, Steven Houben, and Nicolai Marquardt. 2017. EagleSense: Tracking People and Devices in Interactive Spaces Using Real-Time Top-View Depth-Sensing. In Proceedings of the $2017 \mathrm{CHI}$ Conference on Human Factors in Computing Systems (CHI '17). ACM, New York, NY, USA, 3929-3942. DOI : http://dx.doi.org/10.1145/3025453.3025562

[95] Johannes Zagermann, Ulrike Pfeil, Roman Rädle, Hans-Christian Jetter, Clemens Klokmose, and Harald Reiterer. 2016. When tablets meet tabletops: The effect of tabletop size. In Conference on Human Factors in Computing Systems - Proceedings (CHI '16). DOI : http://dx.doi.org/10.1145/2858036.2858224 